\title{
ARE THE STELLAR POPULATIONS IN STARBURSTS, LINERS AND SEYFERT GALAXIES SIMILAR ?
}

\author{
M. JOLY, C. BOISSON, D. PELAT
}

$D A E C$

Observatoire de Meudon F-92195 Meudon

M. SEROTE ROOS

Observatório Astronómica de Lisboa

Tapada da Ajuda, 1300 Lisboa

AND

M.J. WARD

$X$-ray Astronomy Group, Dept. of Physics

University of Leicester, Leicester LE1 7RH

\section{Introduction}

It is well known that about $10 \%$ of galaxies harbour an Active Galactic Nuclei (AGN). A debated question is whether the activity influences the stellar population, or inversely, whether the level of activity is induced by the stellar population. By inspection of AGN spectra of low activity level, it is obvious that starlight contributes to a substantial fraction of the light.

It is generally admitted that the stellar populations in the central part of galaxies vary following the morphological type, although a very large spread is observed within each type. We demonstrate that, in the case of AGN, the observed variety of populations in the nuclei could be associated mainly with the level of activity rather than with the morphological type of the host galaxy.

The simplest way to determine stellar population is to perform stellar population synthesis of the integrated starlight. That is to compare line equivalent widths observed in galaxies with a linear combination of the equivalent widths of the same lines from a library of observed star spectra. This is the method which requires the smallest number of hypotheses 
(no assumptions regarding the IMF or the evolutionary history of the star formation).

The main problem is the mathematical method. We are looking for the contribution of each stellar type to the total radiation. We have thus to solve a system of non-linear equations with the constraint that the contribution must be positive. Up to now, methods do not include directly this positivity constraint and did not deliver a unique solution. A new method has been developed (Pelat, 1997 MNRAS 284, 365) which includes the positivity constraint and determines an approximate but unique solution. When used on test data the method has been shown to converge towards the true population, as the $\mathrm{S} / \mathrm{N}$ of the spectra increases."

We performed long slit spectroscopy of a sample of galaxies from various classes of activity in the range $5000-10000 \AA$. This range is relatively free of emission lines and rich in stellar signatures. About 40 stellar features in the spectra of the galaxies and of a data base of 26 stars were measured.

\section{Results}

-Starburst. As expected, hot stars largely dominate the radiation in the visible range: $\sim 80 \%$ of the light at $\lambda=5450 \AA$. The metallicity is about solar and the internal reddening is high. This is characteristic of a recent burst of star formation in the nucleus $\left(\leq 10^{6} \mathrm{Yrs}\right)$.

-Seyfert 2. A hot component is radiating $\sim 40 \%$ of the light. Metallicity is higher than solar. Internal reddening is high. This is indicative of a recent but weaker burst of star formation than for starburst galaxies.

-Seyfert 1. Because of the numerous emission lines, it was not possible to measure enough stellar features in the nucleus. We concentrate on the circum-nuclear regions $(\sim 300-500 \mathrm{pc})$. High metallicity evolved stars are the main contributor to the radiation $(\sim 50 \%)$. The population is more evolved than in the circum-nuclear regions of the Seyfert $2 \mathrm{~s}$ which is again different from the starburst galaxies.

-LINER. Radiation is dominated by the lower main sequence stars $(\geq$ $70 \%$ ), metallicity is high and little internal reddening is observed. This is characteristic of an old stellar population.

In conclusion, stellar populations in the inner 5 arcsec of AGN host galaxies are very different from one class to the other.

Most important is the indication of an age sequence. First, the Starbursts where an intense burst of star formation is occuring. Second, the Seyfert 2s with a weaker/older burst of star formation, plus an old high metallicity component. Then, the Seyfert 1 s with a metal rich evolved population like in a normal galaxy. And finally, the LINERs with an old metal rich population. 